Tropical Journal of Pharmaceutical Research April 2020; 19 (4): 811-815

ISSN: $1596-5996$ (print); 1596-9827 (electronic)

(C) Pharmacotherapy Group, Faculty of Pharmacy, University of Benin, Benin City, 300001 Nigeria.

\title{
Effects of ginsenoside Rg3 on bone loss, bone mineral density and osteoclast number in glucocorticoid-induced osteoporosis rats, and the likely mechanism of action
}

\author{
Maoxiu Peng, Gangyi Jiang, Shaoqi He, Chengxuan Tang, Xiaojun Tang* \\ Department of Orthopaedics, The Third Affiliated Hospital of Wenzhou Medical University, Wenzhou, PR China
}

*For correspondence: Email: ek046q@163.com

Sent for review: 7 December 2019

Revised accepted: 20 January 2020

\begin{abstract}
Purpose: To investigate the effect of ginsenoside Rg3 on bone loss, bone mineral density (BMD) and osteoclast number in glucocorticoid-induced osteoporosis (GIOP) rats, and the mechanism of action involved.

Methods: Sixty female Wistar rats were assigned to control, model group, ginsenoside Rg3, and alendronate sodium groups, comprised of 15 rats per group. The osteoporosis rat model was established via intramuscular injection of dexamethasone. Changes in bone mineral content (BMC), $B M D$ trabecular thickness and area, osteoblasts and osteoclasts in femurs and lumbar vertebrae were measured after 3 months of treatment.

Results: There were significantly higher BMC and BMD levels in ginsenoside Rg3 group than in alendronate rats $(p<0.05)$. The thickness and trabecular area in femur and lumbar vertebrae in the ginsenoside Rg3 group were significantly higher than those in the model group $(p<0.05)$, but were comparable with those in the alendronate sodium group $(p>0.05)$. There were marked increases in osteoblasts, and marked decreases in osteoclasts in the ginsenoside Rg3 group, alendronate sodium and control rats, relative to model rats $(p<0.05)$.

Conclusion: Ginsenoside Rg3 arrests bone loss, and enhances bone density, trabecular thickness and area, bone microstructure, osteoblast activity and population of osteoclasts number in glucocorticoidinduced osteoporotic rats. This provides a new research direction for the clinical treatment of osteoporosis.
\end{abstract}

Keywords: Ginseng soap, Rg3, Glucocorticoid, Osteoporosis, Bone loss, Bone mineral density, Osteoclast population

\begin{abstract}
This is an Open Access article that uses a fund-ing model which does not charge readers or their institutions for access and distributed under the terms of the Creative Commons Attribution License (http://creativecommons.org/licenses/by/4.0) and the Budapest Open Access Initiative (http://www.budapestopenaccessinitiative.org/read), which permit unrestricted use, distribution, and reproduction in any medium, provided the original work is properly credited.

Tropical Journal of Pharmaceutical Research is indexed by Science Citation Index (SciSearch), Scopus, International Pharmaceutical Abstract, Chemical Abstracts, Embase, Index Copernicus, EBSCO, African Index Medicus, JournalSeek, Journal Citation Reports/Science Edition, Directory of Open Access Journals (DOAJ), African Journal Online, Bioline International, Open-J-Gate and Pharmacy Abstracts
\end{abstract}

\section{INTRODUCTION}

Glucocorticoids (GCs) are often used in severe infections or autoimmune diseases and respiratory diseases. Their complex pharmacological effects determine the severity and diversity of their adverse reactions [1]. Glucocorticoid-induced osteoporosis (GIOP) is a is frequently seen in clinics. Systemic metabolic osteopathy characterized by decreased bone strength, increased risk of fracture and bone brittleness may lead to high incidence of serious 
bone pain, osteonecrosis, joint surface collapse and arthritis which seriously affect standard of health [2].

Some studies have found that traditional Chinese medicine effectively relieves pain and improves standard of health and comfort of GIOP sufferers, with fewer toxicity and side effects. Ginseng saponins in some Chinese herbal medicines are the main active components extracted from ginseng plant. They have antiinflammatory, antioxidant, anti-stress and immunomodulatory effects. Several kinds of saponins have been isolated from ginseng, among which Rg3 is considered to be the most physiologically active monomer $[3,4]$.

In this study, a rat model of GIOP was established via super-physiological dose of dexamethasone. Using alendronate sodium as positive control, the effects of ginsenosides Rg3 on bone loss, bone mineral density and osteoclast number in GIOP rats were investigated, with a view to providing experimental basis for clinical prevention and treatment of the disease.

\section{EXPERIMENTAL}

\section{Materials and instruments}

Sixty female, non-pregnant Wistar rats aged 4 months, with mean weight of $200 \pm 20 \mathrm{~g}$, were used. Dexamethasone injection was produced by Guizhou Guangzheng Pharmaceutical Co. Ltd. Ginsenoside Rg3 was purchased from China Aladdin Biochemical Technology Co. Ltd, while alendronate sodium tablets were product of Hainan Mankexin Pharmaceutical Co. Ltd. Automatic Image Digital Analyzer was made by Leica Company, Germany. Luner DPX-MD dualenergy X-ray bone mineral density measuring instrument was purchased from CE Company.

Approval for this study was received from the Animal Ethical Committee of Department of Orthopaedics, The Third Affiliated Hospital of Wenzhou Medical University, Wenzhou City, China (approval no. 201833156). The study was carried out in line with "Principles of Laboratory Animal Care" (NIH, 1985) [5].

\section{Animal grouping and feeding}

The rats in each group were housed in an environment of temperature $19 \pm 3{ }^{\circ} \mathrm{C}$ and humidity $58 \pm 12 \%$. After 15 days of acclimatization on feed and drinking water ad libitum, the rats were randomly divided into control group, model group, ginsenoside Rg3 treatment group and alendronate treatment group, with 15 animals in each group.

\section{Establishment of GIOP rat model}

The model, the ginsenoside $\mathrm{Rg} 3$, and the alendronate groups were intramuscularly injected with dexamethasone twice a week, at a dose of $0.2 \mathrm{mg} / 100 \mathrm{~g}$ body weight, for a total of 12 weeks. The control rats received physiological saline in place of drug.

The rats in ginsenoside $\mathrm{Rg} 3$ treatment group were given ginsenoside Rg3. Rats in the alendronate sodium treatment group were given alendronate sodium suspension at a dose of 10 $\mathrm{mg} / \mathrm{kg}$ body weight via gavage once a day, after a 30-min fast. The course of treatment was 3 months. Rats in the model and control groups were given saline via gavage.

\section{Specimen collection and measurements}

Following anesthesia (diethyl ether), BMD and BMC of lumber spine and left femur (LSLF) of the rats were measured using Lunar DPX-MD Bone mineral density instrument and Small Animal Cascade Standard Analysis software. After BMD measurement, the rats were sacrificed via cardiac puncture. The left femur and the 4th lumbar spine of the rats were quickly removed, and the attached muscles and connective tissue were cut off. The samples were soaked in $75 \%$ alcohol and stored at $-20{ }^{\circ} \mathrm{C}$. The bone specimens were decalcified, dehydrated, embedded and sectioned for routine hematoxylin and eosin (H \& E) staining.

An automatic image digital analyzer was used to analyze the H\&E-stained section images. Five fields of view were randomly selected from each section under a 100-fold optical microscope, and 5 sites were selected from each field of view for measurement of the thickness and area of trabecular bone through mouse segmentation. In addition, five fields of view were randomly selected from each section under a 400-fold optical microscope, and the numbers of osteoblasts and osteoclasts per unit area were calculated with the mouse click method.

\section{Statistical analysis}

Measurement data are expressed as mean \pm standard deviation (SD). Enumeration data are expressed as [n (\%)]. Comparison between groups was done with $t$-test, while multiple groups were compared with Chi-square test. All statistical analyses were done with SPSS 19.0. 
values of $p<0.05$ were taken as indicative of significant differences.

\section{RESULTS}

\section{Changes in BMC and BMD of rat femur}

After 3 months of treatment, BMC and BMD levels in the model group were significantly lower than those in the control, ginsenoside Rg3, and alendronate groups $(p<0.05)$, while BMD was markedly higher in the ginsenoside Rg3 group than in alendronate rats $(p<0.05$; Table 1$)$.

Table 1: Changes in BMC and BMD levels in rat femur $(\mathrm{N}=15)$

\begin{tabular}{|c|c|c|}
\hline Group & BMC (g/kg) & $\begin{array}{c}\text { BMD } \\
\left(\mathrm{g} / \mathrm{cm}^{2} / \mathrm{kg}\right)\end{array}$ \\
\hline Control & $\begin{array}{l}1.478 \pm \\
0.074^{*}\end{array}$ & $\begin{array}{c}0.847 \pm \\
0.765^{\star}\end{array}$ \\
\hline Model & $\begin{array}{l}1.136 \pm \\
0.075^{\#}\end{array}$ & $\begin{array}{c}0.578 \pm \\
0.170^{\#}\end{array}$ \\
\hline Rg3 & $\begin{array}{l}1.397 \pm \\
0.132^{*}\end{array}$ & $\begin{array}{c}0.787 \pm \\
0.381^{*}\end{array}$ \\
\hline Alendronate & $\begin{array}{l}1.289 \pm \\
0.077^{*}\end{array}$ & $\begin{array}{l}0.715 \pm \\
0.045^{\star \$}\end{array}$ \\
\hline \multicolumn{3}{|c|}{$\begin{array}{l}\text { Data are mean } \pm S D ;{ }^{*} p<0.05 \text {, vs model rats; }{ }^{\#} p< \\
0.05 \text {, vs control rats; }{ }^{\$} p<0.05 \text {, vs alendronate rats }\end{array}$} \\
\hline
\end{tabular}

After 3 months of treatment, the thickness and area of the trabeculae of femur and lumbar vertebrae in the ginsenoside Rg3 group were significantly higher than those in the model group $(p<0.05)$, but the difference was not statistically significant when compared with those of the alendronate group $(p>0.05)$, as shown in Table 2. The results of H\&E procedure revealed that control rat trabeculae were wide in thickness, small in spacing and closely arranged, indicating normal trabeculae structures (Figures $1 \mathrm{~A}$ and B).

Model rat trabeculae were evidently thinner, with wide spacing and sparse arrangement, and they were fractured.

The connections between the bone trabecular structures in the ginsenoside $\mathrm{Rg} 3$ group and the alendronate group were reticular and similar to normal bone trabecular structures (Figures $3 \mathrm{~A}$ and $\mathrm{B}$; Figure $4 \mathrm{~A}$ and $\mathrm{B}$ ).
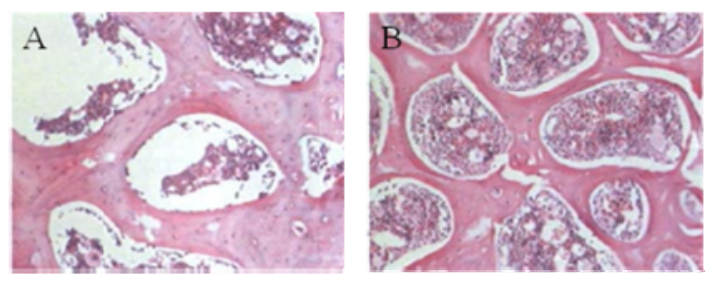

Figure 1: Femoral tissue (A) and lumber tissue (B) morphologies of control group
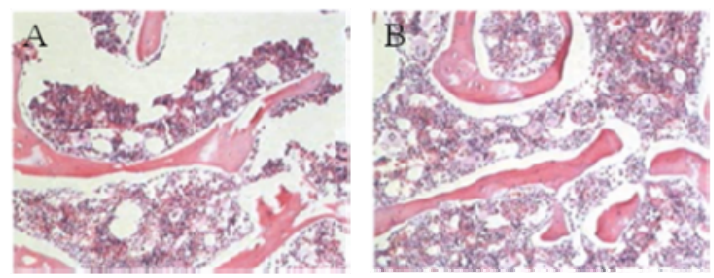

Figure 2: A: Femoral tissue morphology of model group; B: lumbar tissue morphology in model group
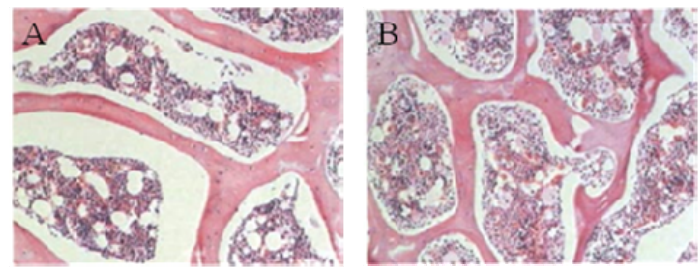

Figure 3: A: Femoral tissue morphology of ginsenoside Rg3 group; $\mathrm{B}$ : lumbar tissue morphology of ginsenoside $\mathrm{Rg} 3$ group
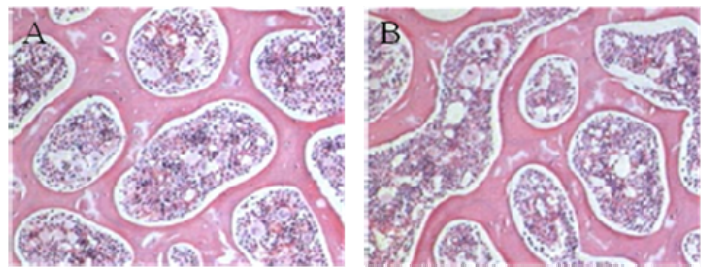

Figure 4: A: Femoral tissue morphology of alendronate group; B: lumbar tissue morphology of alendronate group

Table 2: Changes in the thickness and area of the trabecula of LSLF in each group of rats

\begin{tabular}{lcccc}
\hline \multirow{2}{*}{ Group } & \multicolumn{2}{c}{ Left femur } & \multicolumn{2}{c}{ Lumbar vertebrae } \\
\cline { 2 - 5 } & Thickness $(\boldsymbol{\mu m})$ & Area $\left(\boldsymbol{\mu m ^ { 2 } )}\right.$ & Thickness $(\boldsymbol{\mu m})$ & $\boldsymbol{A r r e a}^{*} \boldsymbol{\mu m ^ { 2 } )}$ \\
\hline Control & $0.375 \pm 0.038^{*}$ & $2.300 \pm 0.347^{*}$ & $0.213 \pm 0.004^{*}$ & $1.627 \pm 0.115^{*}$ \\
Model & $0.138 \pm 0.033^{\#}$ & $0.445 \pm 0.128^{\#}$ & $0.091 \pm 0.002^{\#}$ & $0.541 \pm 0.065^{\#}$ \\
Rg3 & $0.365 \pm 0.035^{*}$ & $1.878 \pm 0.210^{*}$ & $0.179 \pm 0.016^{*}$ & $1.402 \pm 0.089^{*}$ \\
Alendronate & $0.383 \pm 0.031^{*}$ & $1.985 \pm 0.203^{*}$ & $0.187 \pm 0.017^{*}$ & $1.418 \pm 0.101^{*}$ \\
\hline Data are mean \pm SD. ${ }^{*} p<0.05$, vs model rats; ${ }^{*} p<0.05$, vs control rats
\end{tabular}


Table 3: Changes in the number of osteoblasts and osteoclasts in rats

\begin{tabular}{lcccc}
\hline \multirow{2}{*}{ Group } & \multicolumn{2}{c}{ Left femur } & \multicolumn{2}{c}{ Lumbar vertebrae } \\
\cline { 2 - 5 } Control & Osteoblasts & Osteoclasts & Osteoblasts & Osteoclasts \\
\cline { 2 - 5 } Model & $9.675 \pm 1.113^{*}$ & $1.476 \pm 0.517^{*}$ & $9.012 \pm 1.061^{*}$ & $1.602 \pm 0.517^{*}$ \\
Rg3 & $2.410 \pm 0.738^{\#}$ & $5.471 \pm 0.925^{\#}$ & $2.543 \pm 0.641^{\#}$ & $4.756 \pm 0.917^{\#}$ \\
Alendronate & $7.274 \pm 1.534^{* \#}$ & $1.571 \pm 0.901^{*}$ & $6.921 \pm 1.100^{* \#}$ & $1.635 \pm 0.414^{*}$ \\
\hline
\end{tabular}

Data are mean \pm SD. ${ }^{*} p<0.05$, vs model rats; ${ }^{*} p<0.05$, vs control rats

\section{Changes in the number of osteoblasts and osteoclasts in rats}

After 3 months of treatment, there were marked increases in osteoblasts and marked decreases in osteoclasts in the ginsenoside Rg3 group, alendronate sodium and control rat groups, relative to model rats $(p<0.05)$. However, populations of osteoclasts and osteoblasts were comparable between the alendronate sodium and the ginsenoside $\mathrm{Rg} 3$ rat groups $(p>0.05$; Table 3).

\section{DISCUSSION}

Osteoporosis is a systemic skeletal disease that is associated with fracture due to reduction in bone mass and degeneration of bone microstructure, resulting in increased bone brittleness. Studies have shown that bone loss occurs very rapidly within 3-6 months of glucocorticoid treatment, and with increase in GCS dose, bone loss is also increased, thereby complicating GIOP. Several mechanisms are involved in the induction of osteoporosis by GCS $[6,7]$.

Under the action of GCS, parathyroid gland secretion increases, activating osteoclast bone absorption, leading to decreased bone mass. Moreover, GCS inhibits the gastrointestinal absorption of calcium, thereby inhibiting bone formation and promoting bone resorption. Glucocorticoids directly inhibit the production of osteoblasts and the expression of insulin-like growth factor, and reduce bone conversion. They inhibit estrogen secretion, reduce the content of calcium and phosphorus in bone, and induce abnormal lipid metabolism, resulting in the formation of bone marrow microfat plug and bone necrosis.

The current treatment methods for osteoporosis involve external drug intervention with bone resorption inhibitors and bone formation promoters. However, these traditional drugs have many side effects as well as limitations in efficacy [8]. In this study, ginsenoside Rg3 was used to treat GIOP rats, with good results. Bone density determines $70 \%$ of bone strength, and it is an important index for clinical diagnosis of osteoporosis. It is also of great significance for predicting fracture risk. Human bone mineral content is closely related to bone strength and the stability of the internal environment, and plays an important role in the assessment and study of bone physiology and pathology as well as diseases affecting various organs $[9,10]$.

In this study, dexamethasone was used to establish a rat model of GIOP, and the ginsenoside $\mathrm{Rg} 3$ group was simultaneously gavaged with ginsenoside Rg3 group. After 3 months of treatment, BMC and BMD levels of ginsenoside $\mathrm{Rg} 3$ group were similar to those of the control group, and better than those of alendronate group. Compared with the other 3 groups, BMC and BMD levels in the model group were the lowest. These results indicate that ginsenoside $\mathrm{Rg} 3$ mitigated dexamethasoneinduced reduction of bone mineral content and bone density in femur and lumbar vertebrae of rats, promoted mineral deposition in bone, and prevented further loss of bone minerals. These findings are consistent with those of Li et al [11]. Ginsenoside Rg3 increased the thickness and area of trabecular bone and reversed the destruction of bone microstructure in rats possibly through a mechanism involving inhibition of bone resorption, promotion of bone formation, and promotion of the synthesis of estradiol and calcitonin [12].

Osteoblasts function in bone formation, and are responsible for the synthesis and secretion of bone matrix. In contrast, osteoclasts are responsible for bone resorption. The balance between the levels of osteoclasts and osteoblasts is the key to maintenance of normal bone mass $[13,14]$. The results of this study showed that, after 3 months of administration, the numbers of osteoblasts and osteoclasts in the Rg3 group, alendronate group and control group were significantly increased, while the populations of osteoclasts and osteoclasts in the Rg3 group were not significantly different from those in the model group. These findings suggest that ginsenoside $\mathrm{Rg} 3$ reduces bone loss by inhibiting osteoclast formation, differentiation and bone resorption. A similar finding has been reported [15]. 


\section{CONCLUSION}

Ginsenoside Rg3 prevents glucocorticoidinduced bone loss, and at the same time, increases the thickness and area of trabecular bone of rats, and suppresses the destruction of bone microstructure by enhancing bone formation and inhibiting bone resorption. Thus, ginsenoside Rg3 has a potential for use in the development of new drugs for treating osteoporosis.

\section{DECLARATIONS}

\section{Conflict of interest}

No conflict of interest is associated with this work.

\section{Contribution of authors}

We declare that this work was done by the author(s) named in this article and all liabilities pertaining to claims relating to the content of this article will be borne by the authors. All authors read and approved the manuscript for publication. Xiaojun Tang conceived and designed the study, Maoxiu Peng, Gangyi Jiang, Shaoqi He, Chengxuan Tang, Xiaojun Tang collected and analysed the data while Maoxiu Peng wrote the manuscript.

\section{Open Access}

This is an Open Access article that uses a funding model which does not charge readers or their institutions for access and distributed under the terms of the Creative Commons Attribution License (http://creativecommons.org/licenses/by/ 4.0) and the Budapest Open Access Initiative (http://www.budapestopenaccessinitiative.org/rea d), which permit unrestricted use, distribution, and reproduction in any medium, provided the original work is properly credited.

\section{REFERENCES}

1. Anderson EM, McFadden $L M$, Matuszewich L. Interaction of stress and stimulants in female rats: Role of chronic stress on later reactivity to methamphetamine. Behav Brain Res 2019; 376: 112176.

2. Nijland $M$, Boslooper $K$, van Imhoff $G$, Kibbelaar $R$, Joosten $P$, Storm $H$, van Roon EN, Diepstra A, KluinNelemans HC, Hoogendoorn M. Relapse in stage I(E) diffuse large B-cell lymphoma. Hematol Oncol 2018; 36: 416-421.
3. Yang JL, Hu ZF, Zhang TT, Gu AD, Gong T, Zhu P. Progress on the Studies of the Key Enzymes of Ginsenoside Biosynthesis. Molecules 2018; 23(3): 589.

4. Yang J, Zhang JN, Chen WL, Wang GS, Mao Q, Li SQ, Xiong WH, Lin YY, Ge JW, Li XX, Gu Z, et al. Effects of $A Q P 5$ gene silencing on proliferation, migration and apoptosis of human glioma cells through regulating EGFR/ERK p38 MAPK signaling pathway. Oncotarget 2017; 8: 38444-38455.

5. World Health Organization. Principles of laboratory animal care. WHO Chron 1985; 39: 51-56.

6. Nguyen DM, Yassa MA, Tustison NJ, Roberts JM, Kulikova A, Nakamura A, Ivleva El, Van Enkevort E, Brown ES. The Relationship Between Cumulative Exogenous Corticosteroid Exposure and Volumes of Hippocampal Subfields and Surrounding Structures. J Clin Psychopharmacol 2019; 39: 653-657.

7. McNerney MW, Sheng T, Nechvatal JM, Lee AG, Lyons $D M$, Soman S, Liao CP, O'Hara R, Hallmayer J, Taylor $J$, et al. Integration of neural and epigenetic contributions to posttraumatic stress symptoms: The role of hippocampal volume and glucocorticoid receptor gene methylation. PLoS ONE 2018; 13(2): 192222.

8. Yan JG, Duan WH. Research progress of osteoporosis drugs. China Med Review 2015; 12: 34-37.

9. Tanaka S, Yoshida A, Kono S, Ito M. Effectiveness of monotherapy and combined therapy with calcitonin and minodronic acid hydrate, a bisphosphonate, for early treatment in patients with new vertebral fractures: An open-label, randomized, parallel-group study. J Orthop Sci 2017; 22: 536-541.

10. Chen C. Relationship between vitamin D, vitamin B12 and homocysteine levels and bone mineral density in postmenopausal women. Chin J Osteoporos 2019; 25 : 23.

11. Li ZD, Zhao RH, Zhang ZC, Yu J, Gu W, He S, Cao GH. Advances in Biosynthesis and Regulation Mechanism of Notoginseng Saponins. Chin J Exp Tradit Med Formulae 2018; 24: 207-213.

12. Chen SX, Chen FY. Clinical efficacy of bone peptide for injection combined with alendronate sodium in the treatment of osteoporosis. Chin J Mod Drug Appl 2015; 15: 16-18.

13. Liu M, Fan FJ, Shi PJ, Tu ML, Yu CP, Du M. Effects of Different Concentrations of Bovine Lactoferrin on Osteoblast/Osteoclast Co-cultures Effects of Different Concentrations of Bovine Lactoferrin on Osteoblast/Osteoclast Co-cultures. Food Res Development 2017; 38: 1-6.

14. Mo GY, Zhang SC, Li YX, Guo HZ, Guo DQ, Li DX, Tang YC, Mo L, Luo PJ, Ma YH. Establishment of a mouse osteoclast culture system in Transwell laboratory. Bone Injury China 2018; 31: 241-247.

15. Mao Y, Zhang ZR. Effects of ginsenoside-induced bone marrow mesenchymal stem cells on liver regeneration and Wnt/ $\beta$-catenin signaling in rats with acute liver failure. Chin J Clin Pharmacol Ther 2019; 24: 503-510. 